University of Nebraska - Lincoln

DigitalCommons@University of Nebraska - Lincoln

U.S. Department of Veterans Affairs Staff

Publications

U.S. Department of Veterans Affairs

2012

\title{
Proteasome activity and autophagosome content in liver are reciprocally regulated by ethanol treatment
}

\author{
Paul G. Thomes \\ pthomes@unmc.edu \\ Casey S. Trambly \\ University of Nebraska Medical Center \\ Geoffrey M. Thiele \\ University of Nebraska Medical Center, gthiele@unmc.edu \\ Michael J. Duryee \\ University of Nebraska Medical Center, mduryee@unmc.edu \\ Howard S. Fox \\ University of Nebraska Medical Center, hfox@unmc.edu \\ See next page for additional authors
}

Follow this and additional works at: https://digitalcommons.unl.edu/veterans

Thomes, Paul G.; Trambly, Casey S.; Thiele, Geoffrey M.; Duryee, Michael J.; Fox, Howard S.; Haorah, James; and Donohue, Terrence M. Jr, "Proteasome activity and autophagosome content in liver are reciprocally regulated by ethanol treatment" (2012). U.S. Department of Veterans Affairs Staff Publications. 81.

https://digitalcommons.unl.edu/veterans/81

This Article is brought to you for free and open access by the U.S. Department of Veterans Affairs at DigitalCommons@University of Nebraska - Lincoln. It has been accepted for inclusion in U.S. Department of Veterans Affairs Staff Publications by an authorized administrator of DigitalCommons@University of Nebraska - Lincoln. 


\section{Authors}

Paul G. Thomes, Casey S. Trambly, Geoffrey M. Thiele, Michael J. Duryee, Howard S. Fox, James Haorah, and Terrence M. Donohue Jr 


\title{
Proteasome activity and autophagosome content in liver are reciprocally regulated by ethanol treatment
}

\author{
Paul G. Thomes ${ }^{b, *}$, Casey S. Trambly ${ }^{b}$, Geoffrey M. Thiele ${ }^{\mathrm{a}, \mathrm{b}}$, Michael J. Duryee ${ }^{\mathrm{b}}$, Howard S. Fox ${ }^{\mathrm{c}}$, \\ James Haorah ${ }^{c}$, Terrence M. Donohue Jr. ${ }^{\text {a,b }}$ \\ ${ }^{a}$ Liver Study Unit, Dept of Veterans Affairs, Nebraska-Western Iowa Health Care System (N-WIHCS), University of Nebraska Medical Center, Omaha, NE, United States \\ ${ }^{\mathrm{b}}$ Department of Internal Medicine, University of Nebraska Medical Center, Omaha, NE, United States \\ ${ }^{\mathrm{c}}$ Department of Pharmacology and Experimental Neuroscience, University of Nebraska Medical Center, Omaha, NE, United States
}

\section{A R T I C L E I N F O}

\section{Article history:}

Received 3 November 2011

Available online 28 November 2011

\section{Keywords:}

Alcoholic liver injury

Oxidant stress

Lysosomes

Autophagosomes

Proteolysis

\begin{abstract}
A B S T R A C T
The proteasome and autophagy are two major intracellular protein degradation pathways and the regulation of each by ethanol metabolism affects cellular integrity. Using acute and chronic ethanol feeding to mice in vivo, and precision-cut rat liver slices (PCLS) ex vivo, we examined whether ethanol treatment altered these proteolytic pathways. In acute studies, we gave C57Bl/6 mice either ethanol or phosphate-buffered saline (PBS) by gastric intubation and sacrificed them $12 \mathrm{~h}$ later. PCLS were exposed to 0 or $50 \mathrm{mM}$ ethanol for 12 and $24 \mathrm{~h}$ with or without 4-methylpyrazole (4MP). In chronic studies we pair-fed control and ethanol liquid diets for 4-6 weeks to transgenic mice, expressing the green fluorescent protein (GFP) fused to the autophagic marker, microtubule associated protein-1 light chain 3 (LC3). Acute ethanol administration elevated autophagosomes (AVs), as judged by a 1.5-fold increase in LC3II content over PBS-gavaged control mice. Hepatic proteasome activity was unaffected by this treatment. Compared with controls, ethanol exposure for 12 and $24 \mathrm{~h}$ to PCLS inhibited proteasome activity by 1.5- to 3-fold and simultaneously enhanced AVs by 2- to 5-fold. The decrease in proteasome activity and the rise in AVs both depended on ethanol oxidation as its inhibition by 4-methylpyrazole (4MP) blocked both proteasome inhibition and AV induction. Hepatocytes from mice chronically consuming ethanol exhibited a 1.6-fold decline in proteasome activity, and a 4-fold rise in GFP-LC3 puncta compared with pair-fed control mice. When we exposed hepatocytes from these animals to MG262, a proteasome inhibitor, LC3II puncta per cell further increased 2- to 5-fold over untreated cells. Conclusion: Our findings demonstrate that ethanol metabolism generates oxidants, the levels of which differentially influence the activities of the proteasome and autophagy.
\end{abstract}

(c) 2011 Elsevier Inc. All rights reserved.

\section{Introduction}

The ubiquitin-proteasome system (UPS) is one of the major intracellular proteolytic systems in eukaryotic cells. This proteolytic system, as well as the $20 \mathrm{~S}$ proteasome itself are both considered vital pathways for removing misfolded and damaged proteins [1-3]. Ethanol metabolism generates acetaldehyde and oxidants (e.g. peroxynitrite, lipid peroxides) that likely inhibit the proteasome [4-6], as demonstrated in vivo [7] and in recombinant hepatoma cells that metabolize ethanol [8]. No such inhibition has been observed in cells incapable of ethanol oxidation [8], which has

\footnotetext{
Abbreviations: LC3, microtubule associated protein-1 light chain 3; ADH, alcohol dehydrogenase; CYP2E1, cytochrome P450 2E1; SDS-PAGE, sodium dodecyl sulfate; 4-MP, 4-methylpyrazole; DAPI, (4',6-Diamidine-2'-phenylindole dihydrochloride).

* Corresponding author. Address: Liver Study Unit, Research Service (151), VA Nebraska-Western Iowa Health Care System, University of Nebraska Medical Center, 4101 Woolworth Avenue, Omaha, NE 68105, United States.

E-mail address: pthomes@unmc.edu (P.G. Thomes).
}

prompted us and others [4] to suggest that metabolic byproducts of ethanol oxidation directly interact with and inhibit the proteasome.

Besides the proteasome, macroautophagy (henceforth called autophagy) is the second major proteolytic pathway and the primary hydrolytic pathway that degrades all other cytoplasmic components. Autophagy begins with the formation of a doublemembrane-bound autophagosome (AV), which sequesters portions of cytoplasm and eventually fuses with lysosomes. The latter contain acid hydrolases that catalyze degradation of the AV contents. Autophagy degrades native and oxidized proteins, damaged organelles and lipid droplets to maintain cellular homeostasis, particularly during nutrient starvation and stress [9]. Unlike the proteasome, autophagy is activated by ethanol exposure as a cellular defensive mechanism against oxidant stress. This activation is attributed to mTOR inactivation by reactive oxygen species (ROS) generated by ethanol metabolism, thereby causing increased AV formation [10]. The UPS, the 20S proteasome and autophagy are 
generally thought to act independently, as they each have distinct subcellular locations and substrate specificity. However, recent findings reveal the existence of reciprocal cross-talk between these pathways, such that the loss of proteasome activity is associated with a gain in autophagy and vice versa [11-14]. Here, we sought to determine whether this occurred in liver tissue and cells after ethanol administration. The studies described herein used three distinct ethanol exposure systems in rodent liver to ascertain whether ethanol exposure indeed suppressed proteasome activity while it enhanced AV content, the initial phase of autophagy. Here, we show that both these changes depended upon ethanol oxidation and were indeed reciprocally related. Further, these hydrolytic pathways exhibited differential sensitivities to ethanol and showed evidence that they are mechanistically interlinked.

\section{Materials and methods}

\subsection{Reagents}

Anti-LC3B was purchased from Cell Signaling Technology Inc. (Danvers MA). Anti-CYP2E1 and anti- $\beta$-actin were from Calbiochem (La Jolla, CA) and Sigma Chemical Co. (St. Louis, MO), respectively. Dr. Michael Felder, University of South Carolina provided rabbit anti-mouse anti-ADH. We purchased protease inhibitor cocktail (\# P2714-1BTL), the proteasome substrate, N-succinylleucyl-leucyl-valyl-tyrosyl-7-amino-4-methyl-coumarin ( $\mathrm{N}$-SucLLVY-AMC), 4-methylpyrazole and other specialized reagents from Sigma.

\subsection{Experimental animals}

The Institutional Animal Care and Use Committee of the N-WIHCS approved all animal procedures. We followed the Guidelines for the use and Care of Laboratory Animals published by the National Institutes of Health. For ex vivo experiments, Wistar rats were purchased from Charles River Laboratories (Wilmington, MA) and maintained on Purina rat chow prior to preparing precision-cut liver slices (PCLS). For acute ethanol studies, we obtained female C57Bl/6 mice (3-9 months of age) from the National Cancer Institute, Frederick, MD or from the Jackson Laboratories, Bar Harbor, ME. Absolute ethanol was diluted to $50 \%(\mathrm{v} / \mathrm{v})$ into double strength $(2 \times)$ phosphate-buffered saline (PBS) and administered to conscious mice by gastric intubation at a single dose of $6 \mathrm{~g} / \mathrm{kg}$ body weight using a $3.8 \mathrm{~cm}$ stainless steel gavage needle (Popper \& Sons, Inc., New Hyde Park, NY) attached to a $1 \mathrm{ml}$ syringe. We gave control animals an equivalent volume (per body wt.) of single strength $(1 \times)$ PBS. For chronic studies, we used transgenic mice (obtained from the Riken BioResource Center (Ibaraki, Japan) via Dr. Howard Fox (UNMC) that express green fluorescent protein (GFP) fused to the $\mathrm{NH}_{2}$-terminus of microtubule-associated protein light chain 3 (GFP-LC3). GFP-LC3 protein was constitutively expressed in all tissues and driven by the cytomegalovirus immediate-early enhancer linked to the chicken beta-actin promoter (known as CAG). Mice were paired by weight and fed Lieber-DeCarli control or ethanol diet for 35-42 days, using the 10-day adjustment period, during which the ethanol concentration in the diet was gradually increased [15].

\subsection{Precision-cut liver slices (PCLS)}

PCLS were prepared exactly as described by Klassen et al., 2008 and incubated in Williams Medium E [16] with zero or $50 \mathrm{mM}$ ethanol, (with or without additions) at $37^{\circ} \mathrm{C}$ in a Dynamic Organ Culture incubator for durations indicated in the figures. Following incubation, a sample of media was removed from each vial and analyzed for ethanol and acetaldehyde concentrations [15,17]. Each liver slice was then sonicated in PBS and analyses were conducted.

\subsection{Hepatocyte isolation and microscopy}

Mice were anesthetized by IP injection of Nembutal ${ }^{\mathrm{TM}}$ (50 mg/ $\mathrm{kg}$ body weight) and livers were perfused with collagenase followed by Percoll ${ }^{\mathrm{TM}}$ fractionation [18] to isolate mouse hepatocytes. We seeded hepatocytes onto collagen-IV-coated glass cover slips in Williams E medium. After cell attachment for $1 \mathrm{~h}$, the medium was replaced with fresh Williams $\mathrm{E}$ medium and incubation was continued overnight. We then fixed and mounted the cells in $4 \%$ paraformaldehyde using a Vectashield mounting medium with DAPI. All images were acquired with a Zeiss 510 Meta Confocal Laser Scanning Microscope.

\subsection{Proteasome catalytic assay}

Chymotrypsin-like activity of the $20 \mathrm{~S}$ proteasome was measured by incubating cell and tissue extracts with the fluorogenic peptide substrate, $N$-Suc-LLVY-AMC, using the procedure described previously [19]. Proteasome specific activity units are nmoles AMC per h per mg protein.

\subsection{Lactate dehydrogenase assay}

Enzyme activity in both the medium and cell lysates was measured by the rate of oxidation of $130 \mu \mathrm{M}$ NADH using $670 \mu \mathrm{M}$ pyruvate as the substrate in $100 \mathrm{mM}$ potassium phosphate buffer ( $\mathrm{pH} 7.5$ ) in a $1.5 \mathrm{ml}$ reaction mixture for $2 \mathrm{~min}$. The percent release of LDH was expressed as the units of LDH activity in the medium divided by the sum of the LDH activity units in both the cells and the medium $(100 \times)$ [8].

\subsection{Other assays}

Reduced glutathione was measured in liver homogenates, using the enzymatic recycling technique [20]. We used thiobarbituric acid [21] to measure lipid peroxides, the levels of which are expressed as malondialdehyde (MDA) equivalents per gram of liver.

\subsection{Tissue fractionation}

A portion of mouse liver was homogenized in $0.05 \mathrm{M}$ Tris- $\mathrm{HCl}$, $0.25 \mathrm{M}$ sucrose ( $\mathrm{pH} 7.4$ ) and subjected to differential centrifugation, as previously described [22]. Subcellular fractions were frozen at $-70^{\circ} \mathrm{C}$ until they were used for assays.

\subsection{Quantification of proteins by Western blot}

We separated proteins from cell and tissue lysates on $12 \%$ polyacrylamide gels (SDS-PAGE) and transferred them onto nitrocellulose membranes, which we subsequently incubated with anti-LC3II (Cell Signaling Technology Inc.), anti-CYP2E1 (Calbiochem, La Jolla, CA) or anti-ADH. The membranes were then incubated with either horseradish peroxidase-conjugated- or fluorescent secondary antibodies and developed using enhanced chemiluminescence (ECL) (Amersham) or by Odyssey ${ }^{\mathrm{TM}}$ infrared imaging system (Licor, Inc., Lutz, FL). We densitometrically quantified each immunoreactive protein band using Quantity One software (Bio-Rad, Hercules, CA) or by the Licor analysis software. Protein load was normalized by calculating the densitometric ratio of the protein of interest to that of $\beta$-actin. 


\subsection{Statistical analyses}

Data are mean values \pm SEM. We determined statistical significance between two groups by Student's $t$-test and among multiple groups by one way analysis of variance using Newman-keuls post hoc analysis. A probability value $(P) \leqslant 0.05$ was statistically significant.

\section{Results}

\subsection{Acute ethanol administration enhanced LC3II content in mouse} liver while proteasome activity was unchanged

Fifteen minutes after a single acute gavage with 50\% ethanol serum ethanol levels were $113 \pm 2 \mathrm{mM}$. Twelve hours after treatment the serum ethanol level declined to $6.7 \pm 0.5 \mathrm{mM}$, indicating a rapid ethanol clearance rate in these animals. Ethanol-gavaged mice exhibited no change in hepatic CYP2E1 activity at $12 \mathrm{~h}$ or at any other time (up to $48 \mathrm{~h}$ ) after treatment (data not shown). Serum ALT activity in ethanol-fed mice was the same as in controls (Table 1 ) but livers from ethanol-fed mice exhibited signs of hepatic oxidant stress, judged by a $40 \%$ reduction in hepatic glutathione (GSH) (controls: $52 \pm 4$; EtOH-fed: $30 \pm 5$ nmoles $\mathrm{GSH} / \mathrm{mg}$ protein; $P=0.033 ; N=4$ per group) and a 5.6 -fold rise in lipid peroxides (controls: $283 \pm 37$; EtOH-fed: $1582 \pm 193$ nmoles MDA equiv/g liver $P=0.002 ; N=4$ per group) compared with controls. Despite these latter changes, hepatic proteasome chymotrypsin-like activity was the same (Fig. 1A). However, ethanol administration caused a 1.5-fold rise in liver LC3II content over controls (Fig. 1B). This is consistent with the rise in AVs in mouse liver $16 \mathrm{~h}$ after four equally-divided ethanol gavages spaced 20 min apart, as recently reported [10]. Thus, AV content in liver rose without a decline in proteasome activity $12 \mathrm{~h}$ after a single ethanol dose, clearly indicating that AV formation is more sensitive than the proteasome to ethanol-induced oxidant stress.

\subsection{Ethanol metabolism by PCLS suppressed proteasome activity and enhanced LC3II content}

Compared with control liver slices, exposure of PCLS to $50 \mathrm{mM}$ ethanol caused a 2.7- and 1.5-fold decline in $20 \mathrm{~S}$ proteasome chymotrypsin-like activity and a 5- and 2-fold rise in LC3II content after 12 and $24 \mathrm{~h}$ of incubation, respectively (Fig. 2A and B). Inhibition of ethanol oxidation by 4-methylpyrazole (4MP) blocked the decrease in proteasome activity and the rise in LC3II content (Fig. 2A and B). We confirmed the metabolic blockade by higher residual media ethanol levels of $44 \pm 2$ and $34 \pm 9 \mathrm{mM}$ after 12 and $24 \mathrm{~h}$, respectively compared with media ethanol levels of $32 \pm 2$ and $21 \pm 9 \mathrm{mM}$ without $4 \mathrm{MP} \quad(P=0.002$ at $12 \mathrm{~h}$ and $P=0.019$ at $24 \mathrm{~h}$ ). Furthermore, PCLS treated with $50 \mathrm{mM}$ ethanol

Table 1

ALT levels and LDH leakage after ethanol treatment.

\begin{tabular}{|c|c|c|c|c|}
\hline \multirow[t]{2}{*}{ Model system } & \multicolumn{2}{|c|}{ ALT (units/liter) } & \multicolumn{2}{|c|}{ LDH (\% leakage) } \\
\hline & Control & EtOH & Control & EtOH \\
\hline Acute $^{A}$ & $107 \pm 4.8^{\mathrm{a}}$ & $109 \pm 20.6^{a}$ & ND & ND \\
\hline PCLS $^{\mathrm{B}}$ & ND & ND & $23 \pm 2^{\mathrm{a}}$ & $37 \pm 3^{b}$ \\
\hline Chronic $^{\mathrm{C}}$ & $46 \pm 12^{\mathrm{a}}$ & $97 \pm 14^{b}$ & ND & ND \\
\hline
\end{tabular}

All data are mean values $( \pm \mathrm{SEM})$. Letter symbols have same meaning as in Fig. 1 . Note: $\mathrm{ND}=$ not determined.

A Data from acute ethanol studies are taken from animals (5-7 per group) sacrificed $12 \mathrm{~h}$ after treatment.

${ }^{\text {B }}$ LDH activities were from media and slices (4-8 samples) incubated $24 \mathrm{~h}$.

c Data from chronic ethanol studies are from mice (12-15 per group) subjected to 4-6 weeks of pair-feeding. alone generated $12.5 \mu \mathrm{M}$ acetaldehyde; no acetaldehyde was detected in media of PCLS exposed to ethanol and 4MP. Ethanol toxicity, judged by a 1.6-fold rise in LDH leakage into the media of ethanol-treated PCLS relative to controls was detected after $24 \mathrm{~h}$ of exposure (Table 1). Inclusion of 4MP with $50 \mathrm{mM}$ ethanol completely prevented such leakage ( $16 \pm 6 \%$; NS vs. controls). $4 \mathrm{MP}$ alone did not affect LDH leakage (data not shown). Together, these findings indicate that ethanol metabolism by liver slices caused a decline in proteasome activity and the degree of this decrease was associated with a similar magnitude of increase in LC3 content.

\subsection{Chronic ethanol administration suppressed proteasome activity and enhanced autophagosome content in mouse hepatocytes}

In a third ethanol feeding model, we subjected transgenic GFPLC3 mice to chronic ethanol feeding for 4-6 weeks. Serum ALT activity was elevated in sera of ethanol-fed mice compared with pair-fed control mice (Table 1). Compared with controls, ethanol feeding caused a 1.7-fold decline in proteasome activity in hepatocytes obtained from these animals (Fig. $3 \mathrm{~A}$ ). The reduction in enzyme activity was associated with 2.5 -fold higher levels of both CYP2E1 and ADH proteins over pair-fed controls (Table 2). Confocal microscopy revealed a 4-fold higher number of fluorescent AVs in hepatocytes of ethanol-fed mice than in cells from pair-fed controls (Fig. 3B, left two panels). We tested whether AV numbers were sensitive to additional proteasome inhibition, by treating the hepatocytes overnight with $50 \mathrm{nM}$ MG262. This treatment further enhanced the numbers of GFP-LC3 puncta by 2.5 -fold in cells from controls and by 1.3-fold in hepatocytes from ethanol-fed mice (Fig. 3B, right two panels). Western blot analyses of post-nuclear and isolated mitochondrial/lysosomal $(\mathrm{M} / \mathrm{L})$ fractions from whole liver also showed 5- to 6-fold higher levels of LC3II protein in ethanol-fed mice than in pair-fed controls (Fig. 3C and D). The latter findings indicate a strong association between the loss of proteasome activity and the rise in AVs, which was augmented when cells were treated with MG262.

We determined the correlation between the ethanol-elicited fold-decrease (relative to controls) in proteasome activity to the fold-increase (relative to controls) in LC3II content or AV number in the three ethanol exposure models. Data in Fig. 4 demonstrate a strong correlation coefficient of 0.83 between these two parameters. It will be noted that this plot intersects the ordinate at 1.5 , not at 1 indicating that in the absence of proteasome inhibition, ethanol administration still enhanced AV (LC3II) content, likely due to enhanced AV formation. Thus, based upon these results and the recent finding that the 20S proteasome degrades LC3II [23] our data indicate that the ethanol-induced decline in proteasome activity stabilizes LC3II, thereby elevating its content.

\section{Discussion}

Here we observed that three separate ethanol treatment models in rodent liver caused differing degrees of liver injury, which, in all cases, enhanced AV content but differentially decreased proteasome activity, depending on the extent of tissue injury. Acute ethanol administration to mice caused overt but apparently mild signs of oxidant stress, associated with an increase in AV (LC3II) content, which likely indicated a rise in the rate of autophagic flux (i.e. enhanced AV synthesis [24]). AV induction requires an increased rate of phagophore formation, which engulfs cytoplasmic materials while it elongates and matures into a complete autophagosome [25]. Because the rise in LC3II occurred rapidly (within $12 \mathrm{~h}$ after ethanol administration), it is likely that the swift rate of hepatic ethanol oxidation generated sufficient oxidants to induce the 

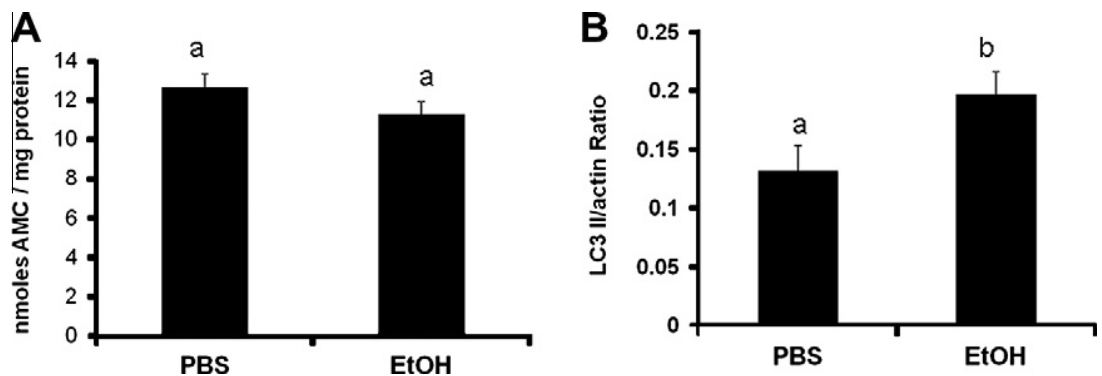

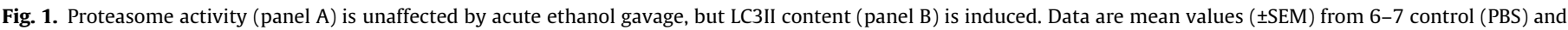
ethanol-gavaged animals $12 \mathrm{~h}$ prior to death. Bars with different alphabets are significantly different from each other. Bars with same alphabet are not.
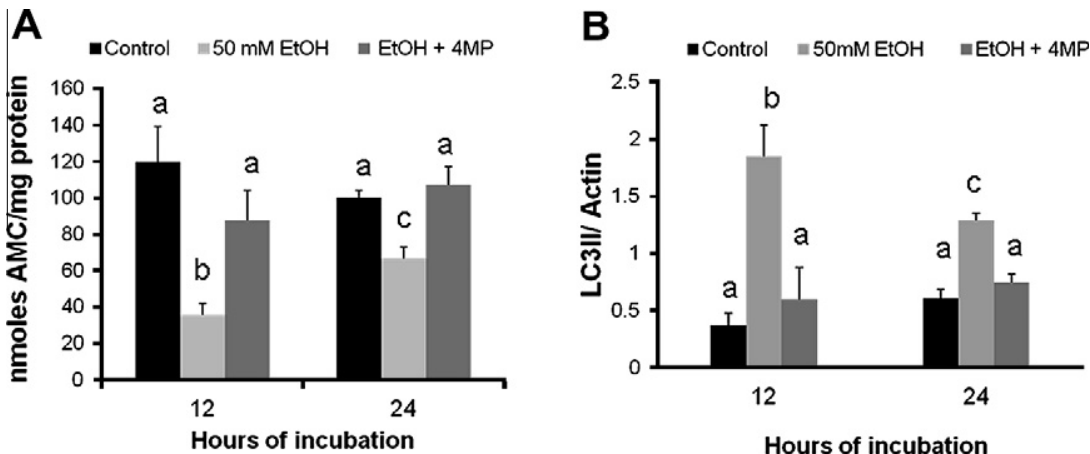

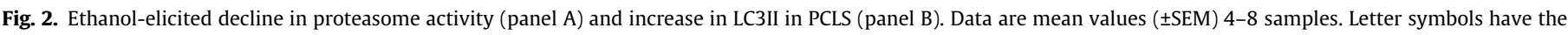
same meaning as in Fig. 1. Note: 4MP data were similar to that of EtOH + 4MP and are not shown.

A

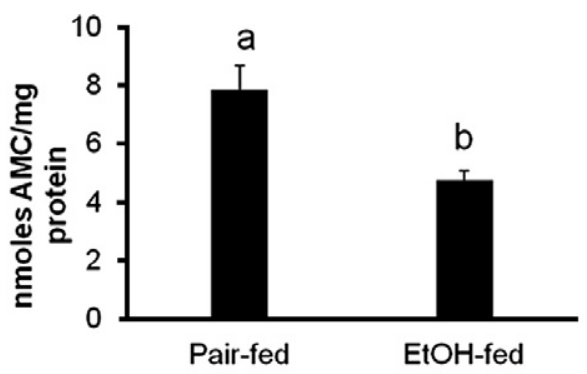

B

Control

EtOH-fed

Control + MG262

EtOH-fed + MG262

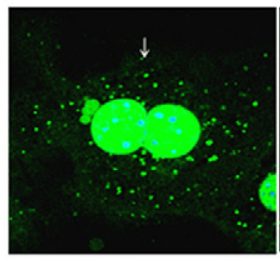

$25 \pm 2^{a}$

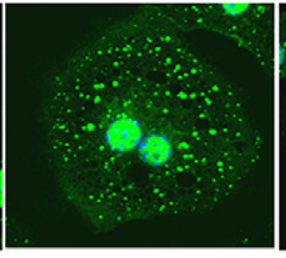

$103 \pm 13^{b}$

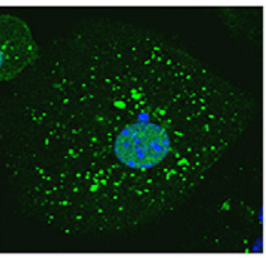

$60 \pm 10^{c}$

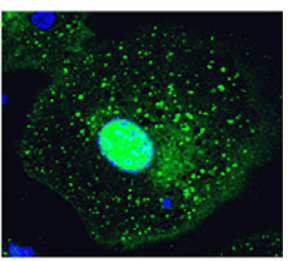

$135 \pm 13^{d}$
C

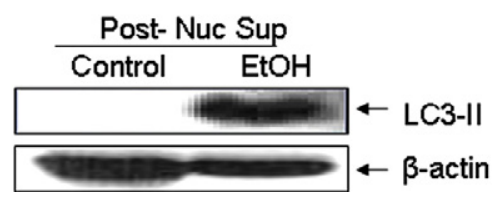

D

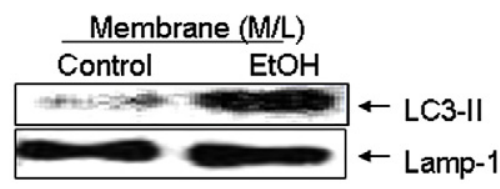

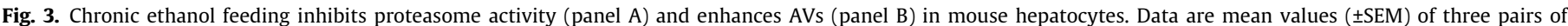

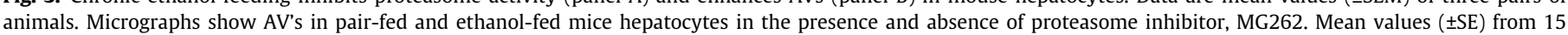

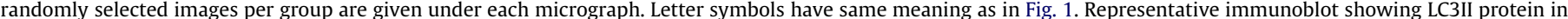

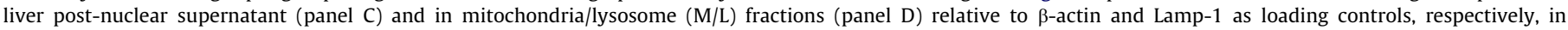
fractionated liver homogenates from pair-fed and EtOH-fed mice. 
Table 2

CYP2E1 and ADH protein levels in mouse hepatocytes after chronic ethanol administration.

\begin{tabular}{lll}
\hline Protein & Pair-fed & EtOH-fed \\
\hline CYP2E1 & $0.9 \pm 0.8^{\mathrm{a}}$ & $2 \pm 0.2^{\mathrm{b}}$ \\
ADH & $0.2 \pm 0.09^{\mathrm{a}}$ & $0.7 \pm 0.16^{\mathrm{b}}$
\end{tabular}

Densitometric ratio of CYP2E1 and ADH protein bands to that of actin. Data are mean values $( \pm$ SEM) of three pairs of animals. Letter symbols above the bars have same meaning as in Fig. 1.

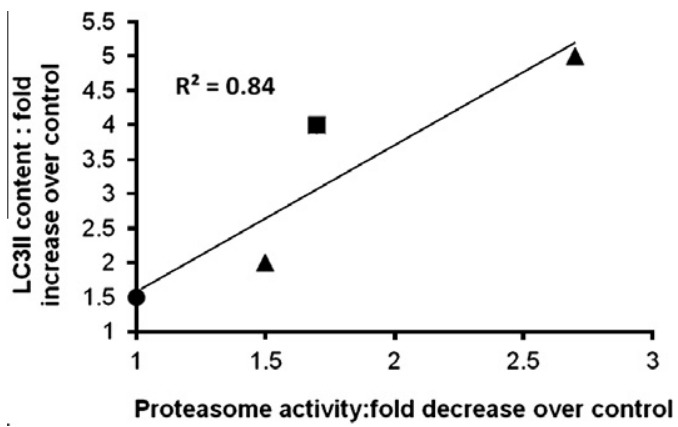

Fig. 4. Correlation between the ethanol-induced decrease in proteasome activity and the ethanol-induced rise in autophagosome content in whole liver after acute ethanol administration, direct treatment with ethanol of PCLS and chronic ethanol administration. A value of 1 means no change in either parameter. Symbols are acute ethanol: solid circle ( $\bullet$ ); PCLS: triangle ( $\boldsymbol{\Delta})$; chronic ethanol feeding $(\boldsymbol{\square})$.

signaling cascade that enhances AV formation as a defensive response [10]. At the same time, acute ethanol treatment left proteasome activity unaffected, probably because higher sustained serum alcohol levels (i.e. $\geqslant 40 \mathrm{mM}$ ) are usually required to achieve inhibition of the enzyme in vivo [26]. It is likely that, along with the rapid rate of ethanol metabolism, the level and/or the duration of oxidant stress was/were insufficient to inhibit the proteasome, as in these acute experiments we observed a restoration of liver GSH levels to normal $24 \mathrm{~h}$ after ethanol gavage (data not shown).

Proteasome inhibition in PCLS after exposure to $50 \mathrm{mM}$ ethanol was consistent with our previous findings, which showed that, high (i.e. 50-100 mM) extracellular ethanol concentrations inhibit $20 S$ proteasome chymotrypsin-like activity in cultured cells [8]. In PCLS, both proteasome inhibition and AV elevation were dependent on ethanol oxidation. In addition, the finding that 4MP blocked both acetaldehyde production and LC3II enhancement indicates that acetaldehyde participated in the elevation of AV content in PCLS. Additionally, in PCLS, inhibition of proteasome activity by $50 \mathrm{mM}$ ethanol was likely due to sustained production of reactive metabolites via $\mathrm{ADH}$ - and CYP2E1-catalyzed ethanol oxidation. Compared with the acute studies just described, the reduction in proteasome activity in PCLS was associated with a greater degree of increase in the content of LC3II. These findings lead us to suggest that in ethanol-exposed PCLS, part of the increase in LC3II was caused by its stabilization, as LC3 is not only degraded in the autolysosome during normal autophagy but is also a target of the 20S proteasome [23], clearly indicating a direct interaction between components of the two degradative systems. Our findings are consistent with recent reports that the two proteolytic pathways are mechanistically interlinked [27]. For example, in response to increased production of misfolded proteins, the UPS is overwhelmed and autophagy is induced as a compensatory mechanism to remove misfolded proteins [28]. Conversely, autophagy inhibition in cultured cardiomyocytes by 3-methyladenine causes enhanced proteasome chymotrypsin-like activity [14] by mechanism(s) that are undefined.
Our findings from ex vivo studies with PCLS were confirmed by similar results in vivo, as chronic ethanol feeding to mice reduced proteasome activity and increased autophagosome content. Proteasome inhibition in hepatocytes after chronic ethanol feeding to GFP-LC3 transgenic mice also coincided with increased CYP2E1 and ADH contents. Both these proteins are proteasome substrates $[29,30]$ and their rise indicated that the metabolites generated from chronic ethanol oxidation likely inhibited not only the $20 \mathrm{~S}$ proteasome but also the 26S proteasome, which is the larger version of the 20S particle and the key degradative component of the UPS that selectively degrades ubiquitylated protein substrates $[29,30]$. Part of the robust increase in autophagosome number after chronic ethanol feeding reflected the decline in proteasome activity coupled with faulty trafficking and degradation of AVs by lysosomes. Further confirmation that the proteasome partially regulates LC3 content was that AV numbers increased in response to overnight treatment of hepatocytes with MG262.

In summary, autophagosomes were elevated in livers of acute ethanol fed mice, in ethanol-exposed PCLS and in livers and hepatocytes of chronically ethanol-fed mice, three widely used ethanol exposure models that exhibit different levels of liver injury. We conclude that the ethanol-elicited rise in AV formation includes activation of AV formation, disruption in AV degradation by lysosomes and now, AV stabilization by ethanol-induced proteasome inhibition. These findings indeed indicate reciprocal regulation of the two proteolytic pathways and provide a cogent explanation for the same observation in other systems [14]. However, this report is unique in that it is one of the first studies to demonstrate that the oxidation of ethanol, a natural liver substrate, generates metabolites that alter the catabolism by the proteasome, of LC3, the most widely-used biomarker of autophagy.

\section{Financial support}

Supported by funds from A Dean's Reviewed Research Grant from the University of Nebraska Medical Center (UNMC) and from internal funds from the Division of Gastroenterology and Hepatology, Department of Internal Medicine, UNMC.

\section{Disclaimer}

The contents of this paper do not represent the views of the Department of Veterans Affairs or the United States Government.

\section{Acknowledgments}

Adam M. Szlachetka, provided valuable technical assistance in this work. Dr. Carol Casey provided technical expertise in liver perfusion and hepatocyte isolation. Drs. Natalia Osna and Abdul Muneer provided helpful scientific input during the course of this work. The data presented here are results of work supported with resources and the use of facilities at the VA Nebraska-Western Iowa Health Care System of the Department of Veterans Affairs, Veterans Health Administration, Office of Research and Development.

\section{References}

[1] T.M. Donohue Jr., The ubiquitin-proteasome system and its role in ethanolinduced disorders, Addict. Biol. 7 (2002) 15-28.

[2] T.M.J. Donohue, The ubiquitin-proteasome system in alcohol-induced pathology, Compr. Handb. Alcohol Relat. Pathol. 2 (2005) 1028-1039.

[3] T.M. Donohue Jr., A.I. Cederbaum, S.W. French, S. Barve, B. Gao, N.A. Osna, Role of the proteasome in ethanol-induced liver pathology, Alcohol Clin. Exp. Res. 31 (2007) 1446-1459.

[4] F. Bardag-Gorce, B.A. French, L. Nan, H. Song, S.K. Nguyen, H. Yong, J. Dede, S.W. French, CYP2E1 induced by ethanol causes oxidative stress, proteasome 
inhibition and cytokeratin aggresome (Mallory body-like) formation, Exp. Mol. Pathol. 81 (2006) 191-201.

[5] F. Bardag-Gorce, J. Li, B.A. French, S.W. French, Ethanol withdrawal induced CYP2E1 degradation in vivo, blocked by proteasomal inhibitor PS-341, Free Radic. Biol. Med. 32 (2002) 17-21.

[6] D.J. Tuma, Role of malondialdehyde-acetaldehyde adducts in liver injury $(1,2)$, Free Radic. Biol. Med. 32 (2002) 303-308.

[7] T.M. Donohue Jr., R.K. Zetterman, Z.Q. Zhang-Gouillon, S.W. French, Peptidase activities of the multicatalytic protease in rat liver after voluntary and intragastric ethanol administration, Hepatology 28 (1998) 486-491.

[8] T.M. Donohue, N.A. Osna, D.L. Clemens, Recombinant Hep G2 cells that express alcohol dehydrogenase and cytochrome P450 2E1 as a model of ethanolelicited cytotoxicity, Int. J. Biochem. Cell Biol. 38 (2006) 92-101.

[9] T.M. Donohue Jr., Autophagy and ethanol-induced liver injury, World J. Gastroenterol. 15 (2009) 1178-1185.

[10] W.X. Ding, M. Li, X. Chen, H.M. Ni, C.W. Lin, W. Gao, B. Lu, D.B. Stolz, D.L. Clemens, X.M. Yin, Autophagy reduces acute ethanol-induced hepatotoxicity and steatosis in mice, Gastroenterology 139 (2010) 1740-1752.

[11] K. Zhu, K. Dunner Jr., D.J. McConkey, Proteasome inhibitors activate autophagy as a cytoprotective response in human prostate cancer cells, Oncogene 29 (2010) 451-462.

[12] V.I. Korolchuk, A. Mansilla, F.M. Menzies, D.C. Rubinsztein, Autophagy inhibition compromises degradation of ubiquitin-proteasome pathway substrates, Mol. Cell 33 (2009) 517-527.

[13] T. Lamark, T. Johansen, Autophagy: links with the proteasome, Curr. Opin. Cell Biol. 22 (2010) 192-198.

[14] P. Tannous, H. Zhu, A. Nemchenko, J.M. Berry, J.L. Johnstone, J.M. Shelton, F.J. Miller Jr., B.A. Rothermel, J.A. Hill, Intracellular protein aggregation is a proximal trigger of cardiomyocyte autophagy, Circulation 117 (2008) 30703078.

[15] T.M. Donohue Jr., T.V. Curry-McCoy, S.L. Todero, R.L. White, K.K. Kharbanda, A.A. Nanji, N.A. Osna, L-Buthionine $(S, R)$ sulfoximine depletes hepatic glutathione but protects against ethanol-induced liver injury, Alcohol Clin. Exp. Res. 31 (2007) 1053-1060.

[16] L.W. Klassen, G.M. Thiele, M.J. Duryee, C.S. Schaffert, A.L. DeVeney, C.D. Hunter P. Olinga, D.J. Tuma, An in vitro method of alcoholic liver injury using precision-cut liver slices from rats, Biochem. Pharmacol. 76 (2008) 426-436.

[17] C.J. Eriksson, H.W. Sippel, O.A. Forsander, The occurrence of acetaldehyde binding in rat blood but not in human blood, FEBS Lett. 75 (1977) 205-208.

[18] S.R. Dalton, R.L. Wiegert, C.R. Baldwin, K.M. Kassel, C.A. Casey, Impaired receptor-mediated endocytosis by the asialoglycoprotein receptor in ethanol- fed mice: implications for studying the role of this receptor in alcoholic apoptosis, Biochem. Pharmacol. 65 (2003) 535-543.

[19] N.A. Osna, R.L. White, S. Todero, B.L. McVicker, G.M. Thiele, D.L. Clemens, D.J. Tuma, T.M. Donohue Jr., Ethanol-induced oxidative stress suppresses generation of peptides for antigen presentation by hepatoma cells, Hepatology 45 (2007) 53-61.

[20] O.W. Griffith, Determination of glutathione and glutathione disulfide using glutathione reductase and 2-vinylpyridine, Anal. Biochem. 106 (1980) 207212.

[21] H. Buttkus, R. Rose, Amine malondialdehyde condensation products and their relative color contribution in the thiobarbituric acid test, J. Am. Oil Chem. Soc. 49 (1972) 440-443.

[22] T.M. Donohue Jr., D.L. McVicker, K.K. Kharbanda, M.L. Chaisson, R.K. Zetterman, Ethanol administration alters the proteolytic activity of hepatic lysosomes, Alcohol Clin. Exp. Res. 18 (1994) 536-541.

[23] Z. Gao, N. Gammoh, P.M. Wong, H. Erdjument-Bromage, P. Tempst, X. Jiang, Processing of autophagic protein LC3 by the 20 S proteasome, Autophagy 6 (2010) 126-137.

[24] D.C Rubinsztein, A.M. Cuervo, B Ravikumar, S. Sarkar, V. Korolchuk, S Kaushik, D.J. Klionsky, In search of an "autophagomometer", Autophagy 5 (2009) 585-589.

[25] D.C. Rubinsztein, G. Marino, G. Kroemer, Autophagy and aging, Cell 146 (2011) 682-695.

[26] T.M. Donohue Jr., K.K. Kharbanda, C.A. Casey, A.A. Nanji, Decreased proteasome activity is associated with increased severity of liver pathology and oxidative stress in experimental alcoholic liver disease, Alcohol Clin. Exp. Res. 28 (2004) 1257-1263.

[27] V.I. Korolchuk, F.M. Menzies, D.C. Rubinsztein, Mechanisms of cross-talk between the ubiquitin-proteasome and autophagy-lysosome systems, FEBS Lett. 584 (2011) 1393-1398.

[28] W.X. Ding, H.M. Ni, W. Gao, T. Yoshimori, D.B. Stolz, D. Ron, X.M. Yin, Linking of autophagy to ubiquitin-proteasome system is important for the regulation of endoplasmic reticulum stress and cell viability, Am. J. Pathol. 171 (2007) 513524.

[29] B.J. Roberts, B.J. Song, Y. Soh, S.S. Park, S.E. Shoaf, Ethanol induces CYP2E1 by protein stabilization. Role of ubiquitin conjugation in the rapid degradation of CYP2E1, J. Biol. Chem. 270 (1995) 29632-29635.

[30] E. Mezey, L. Rennie-Tankersley, J.J. Potter, Liver alcohol dehydrogenase is degraded by the ubiquitin-proteasome pathway, Biochem. Biophys. Res. Commun. 285 (2001) 644-648. 